\section{Preamble Design Using Embedded Signaling for OFDM Broadcast Systems Based on Reduced-Complexity Distance Detection}

\author{
Lifeng He, Zhaocheng Wang, Senior Member, IEEE, Fang Yang, \\ Sheng Chen, Fellow, IEEE, and Lajos Hanzo, Fellow, IEEE
}

\begin{abstract}
The second-generation digital terrestrial television broadcasting standard adopts the so-called P1 symbol as the preamble for initial synchronization. The P1 symbol also carries a number of basic transmission parameters, including the fast Fourier transform size and the single-input/single-output as well as multiple-input/single-output mode, to appropriately configure the receiver for carrying out the subsequent processing. In this paper, an improved preamble design is proposed, where a pair of training sequences is inserted in the frequency domain, and their distance is used for transmission parameter signaling. At the receiver, only a low-complexity correlator is required for the detection of the signaling. Both the coarse carrier frequency offset and the signaling can simultaneously be estimated by detecting the aforementioned correlation. Compared with the standardized P1 symbol, the proposed preamble design significantly reduces the complexity of the receiver while retaining high robustness in frequency-selective fading channels. Furthermore, we demonstrate that the proposed preamble design achieves better signaling performance than the standardized $\mathbf{P 1}$ symbol despite reducing the numbers of multiplications and additions by about $40 \%$ and $20 \%$, respectively.
\end{abstract}

Index Terms-Orthogonal frequency-division multiplexing (OFDM), preamble, second-generation digital terrestrial television broadcasting standard (DVB-T2), transmission parameter signaling (TPS).

\section{INTRODUCTION}

Orthogonal frequency-division multiplexing (OFDM) [1] has found its way into numerous recent standards operating in frequencyselective fading channels. With the aid of inverse fast Fourier transform (IFFT) and fast Fourier transform (FFT) operations, both modulation and demodulation operations of an OFDM system facilitate convenient hardware implementations. Hence, OFDM has widely been adopted in the areas of digital TV [2]-[5], wireless local area networks [1], [6], and next-generation mobile communications [7]-[9]. Multiservice broadcast has become an important research topic in both industry and academia. With the growing commercial demands for supporting multiservices, including HDTV, mobile TV, and data casting, broadcast systems are expected to provide a wide choice of transmission parameters to accommodate different quality-of-service requirements. The European Telecommunications Standards Institute (ETSI) recently issued the second-generation digital terrestrial television broadcasting standard (DVB-T2), which aims for providing multiple services in different propagation scenarios [5].

Manuscript received July 30, 2010; revised October 22, 2010 and December 15, 2010; accepted January 25, 2011. Date of publication February 4, 2011; date of current version March 21, 2011. This work was supported in part by Tsinghua University Initiative Scientific Research Program under Grant 20091081280 and in part by the Standardization Administration of the People's Republic of China (SAC) under AQSIQ Project 200910244. The review of this paper was coordinated by Prof. K. Fukawa.

L. He, Z. Wang and F. Yang are with the Tsinghua National Laboratory for Information Science and Technology, Tsinghua University, Beijing 100084, China (e-mail: hlf04@mails.tsinghua.edu.cn; zcwang@ @singhua.edu.cn; fangyang@tsinghua.edu.cn).

S. Chen and L. Hanzo are with the School of Electronics and Computer Science, University of Southampton, SO17 1BJ Southampton, U.K. (e-mail: sqc@ecs.soton.ac.uk; 1h@ecs.soton.ac.uk).

Color versions of one or more of the figures in this paper are available online at http://ieeexplore.ieee.org.

Digital Object Identifier 10.1109/TVT.2011.2110664
DVB-T2 [5] offers a total of six FFT sizes and seven diverse guard interval modes to adapt to different applications. Furthermore, both single-input/single-output (SISO) and multiple-input/single-output (MISO) transmission modes are supported. Therefore, the efficient and reliable detection of these basic system configuration parameters is critical for the receiver to reliably perform its subsequent processing steps. For this reason, DVB-T2 adopts a specifically designed P1 symbol as the preamble of the DVB-T2 frame. Unlike conventional preambles, which are designed merely for supporting timing and frequency synchronization [10], [11], the P1 symbol also supports the basic transmission parameter signaling (TPS), including the FFT size and the SISO/MISO mode [5]. In the time domain (TD), a novel cyclic extension structure is adopted to improve the peak of the guard interval correlation (GIC) for the sake of improved timing synchronization [12]. In the frequency domain (FD), a length-384 sequence carrying 7-bit signaling is mapped to a distributed subcarrier pattern. To transmit the 7-bit signaling, the standardized P1 symbol of DVB-T2 exploits two sets of orthogonal complementary sequences to represent the different signaling fields, which are known as S1 and S2, respectively. At the receiver, all possible sequences of both sets are correlated with the received signaling sequence to find a matched case. The need to perform such a large number of correlations, however, imposes a high computational complexity on the signaling detection.

In this paper, an improved preamble design is proposed, which carries out the same signaling task as the standardized P1 symbolbased preamble at a lower complexity. Specifically, unlike in the standardized P1 symbol, which incorporates different sequences to convey the signaling information, the proposed preamble inserts a pair of training sequences in the FD, and the signaling information is conveyed by the distance between the pair. At the receiver, only a single correlator is required to simultaneously estimate both the TPS and the coarse carrier frequency offset (CFO); therefore, the complexity of the receiver is significantly reduced. Furthermore, our simulation results show that the proposed preamble design achieves better signaling performance than the standardized P1 symbol-based preamble of the DVB-T2 for transmission over frequency-selective fading channels.

The rest of this paper is organized as follows: Section II briefly describes the standardized P1 symbol in DVB-T2, whereas Section III provides the detailed design of the proposed preamble. Section IV presents our performance evaluation of the proposed preamble design through both theoretical analysis and computer simulation. The computational requirements of detecting both preambles are also compared in this section. Finally, our conclusions are summarized in Section V.

\section{P1 SYMBOL IN DVB-T2}

In this section, we commence with the signal model of OFDM systems and then focus our attention on the P1 symbol design of the DVB-T2 system.

\section{A. Signal Model}

The transmitted TD signal of an OFDM system can be represented as

$$
x_{n}=\frac{1}{\sqrt{N}} \sum_{k=0}^{N-1} X_{k} e^{j \frac{2 \pi}{N} n k}
$$

where $N$ is the number of subcarriers, and $X_{k}$ 's are the transmitted FD data symbols. The received TD OFDM symbol is represented by

$$
y_{n}=x_{n-n_{0}} \otimes h_{n} e^{j 2 \pi f_{c} n}+\nu_{n}
$$




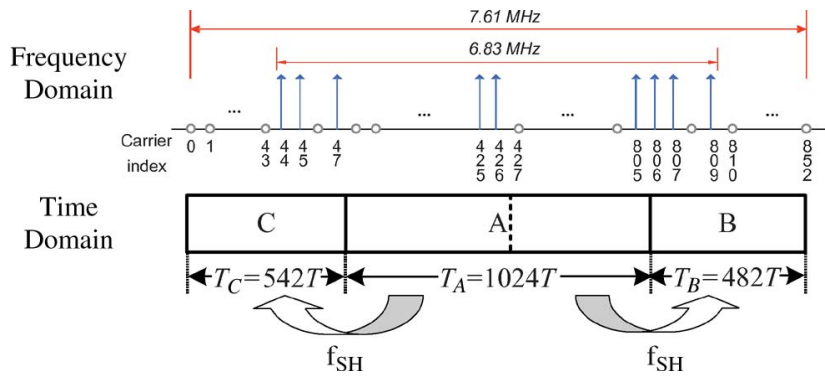

Fig. 1. Structure of the P1 symbol in DVB-T2.

where the operator $\otimes$ denotes linear convolution, $n_{0}$ and $f_{c}$ denote the time delay and CFO, respectively, whereas $h_{n}$ and $\nu_{n}$ denote the channel impulse response and the additive white Gaussian noise (AWGN), respectively. The channel's signal-to-noise ratio (SNR) is defined by $\rho=\sigma_{s}^{2} / \sigma_{n}^{2}$, where $\sigma_{s}^{2}=E\left[\left|x_{n}\right|^{2}\right]$ is the signal power, and $\sigma_{n}^{2}=E\left[\left|\nu_{n}\right|^{2}\right]$ is the AWGN power, with $E[\bullet]$ denoting the statistical expectation operator.

OFDM systems are known to be sensitive to the CFO, which may be separated into two parts as follows:

$$
f_{c}=m_{\mathrm{int}} \cdot \frac{1}{N}+f_{\mathrm{frc}}
$$

where $1 / N$ is the subcarrier spacing, $m_{\text {int }}$ is an integer, and $f_{\text {frc }}$ is the fractional part of the $\mathrm{CFO}$, which is restricted in the range of

$$
-\frac{1}{2 N}<f_{\text {frc }} \leq \frac{1}{2 N}
$$

The integer part of the CFO will lead to a cyclic shift in the FD, whereas the fractional CFO will impose intercarrier interference (ICI), which may severely degrade the attainable performance [13]. In OFDM systems, the training symbols are often transmitted before the data blocks as preambles. The task of the preamble is to accomplish both timing and frequency synchronization as well as to detect the TPS, if it is present.

\section{B. Standardized P1 Symbol}

The P1 symbol defined in the DVB-T2 standard [5] is composed of a 1K OFDM symbol "A" and its two cyclic extensions, which are denoted as "C" and "B", respectively. The C-A-B structure of the $\mathrm{P} 1$ symbol is illustrated in Fig. 1. The TD baseband signal of the P1 symbol is defined by

$$
x_{n}= \begin{cases}p_{A}(n) e^{j 2 \pi f_{\mathrm{SH}} n}, & 0 \leq n<542 \\ p_{A}(n-542), & 542 \leq n<1566 \\ p_{A}(n-1024) e^{j 2 \pi f_{\mathrm{SH}} n}, & 1566 \leq n<2048\end{cases}
$$

where $p_{A}(n)$ is the baseband representation of part "A," and $f_{\mathrm{SH}}$ is the additional frequency shift applied to both parts " $\mathrm{B}$ " and " $\mathrm{C}$ " to distinguish the P1 symbol from the common cyclic prefix of the OFDM symbols.

Out of the 853 useful FD subcarriers of the $1 \mathrm{~K}$ symbol in "A," only 384 subcarriers are used, whereas the others are set to zero. The subcarrier distribution of the P1 symbol is also illustrated in Fig. 1. The active carriers occupy roughly $6.83 \mathrm{MHz}$ in the middle of the nominal 7.61-MHz bandwidth. Even a frequency shift of up to $500 \mathrm{kHz}$ may be estimated, since most of the useful subcarriers are still within the bandwidth. Therefore, the P1 symbol is robust to large CFOs.

The embedded P1 signaling contains two fields, which are referred to as the 3-bit signaling S1 and the 4-bit signaling S2, respectively. Specifically, the 3-bit S1 is represented by one of the eight orthogonal complementary sequences of length 64 , whereas the 4-bit S2 is represented by 1 of the 16 orthogonal complementary sequences of length 256 [5]. The $\mathrm{S} 1$ sequence, the $\mathrm{S} 2$ sequence, and a repetition of the $\mathrm{S} 1$ sequence are concatenated to compose a length-384 signaling sequence, which is first differentially binary phase-shift keying (DBPSK) modulated, then scrambled, and finally mapped to the active subcarriers. It should be noted that a total of $8 \times 16=128$ different signaling sequences correspond to the 7-bit signaling.

\section{Detection of the P1 Symbol}

At the receiver, both timing and fractional CFO estimation may be achieved by the modified GIC method based on the $\mathrm{C}-\mathrm{A}-\mathrm{B}$ structure [12]. After the fractional CFO is compensated, part " $\mathrm{A}$ " of the P1 symbol is extracted and transformed to the FD for integer CFO estimation and signaling detection. First, the energy-detection-based subcarrier pattern matching is performed to locate the exact positions of the active subcarriers by correlating the received signal with the expected carrier distribution sequence (CDS) [14]. The CDS was specifically designed to ensure that only a perfect match gives a sufficiently high power correlation peak. The position of the correlation peak also gives an estimate of the integer part of the CFO, which is inferred from the peak's shift from its original position.

Once the active subcarriers were identified, the receiver is ready to detect the S1 and S2 signaling. The length-384 signaling sequence is first extracted from the active carriers, then descrambled, and differentially decoded. Finally, the 3-bit S1 and 4-bit S2 segments are separated from the signaling sequence. Each legitimate sequence of the $\mathrm{S} 1$ set is correlated with the received $\mathrm{S} 1$ signaling sequence one by one, and the sequence with the largest correlation peak is used for decoding the S1 signaling. Similarly, each legitimate sequence of the $\mathrm{S} 2$ set is correlated with the received S2 signaling sequence one by one to decode the $\mathrm{S} 2$ signaling. Since there are a total of eight sequences in the $\mathrm{S} 1$ set and 16 sequences in the $\mathrm{S} 2$ set, a large number of correlations are required. To reduce the complexity of computing such a large number of correlations, DVB-T2 adopted a series of complementary sets of sequences (CSS) [15]. These correlations may be determined with the aid of efficient correlators specifically designed for CSS [16]. However, the total computational complexity of the signaling detection remains high due to the specific design of the P1 symbol, which will be further discussed in Section IV.

\section{Design of the Proposed Preamble}

In this section, we propose an improved preamble relying on a different signaling design, which is significantly less complex to decode.

\section{A. Structure of the Proposed Preamble}

The structure of the proposed preamble is shown in Fig. 2, which inherits the structure of the standardized P1 symbol in the TD, whereas in the FD, the TPS design is different from that of the P1 symbol. Specifically, a pair of FD training sequences, which is denoted by $(a, b)$, is inserted into the subcarriers. The FD values $\left\{X_{k}\right\}_{k=0}^{N-1}$ of part "A" in the proposed preamble are represented by

$$
X_{k}=\left\{\begin{array}{lc}
a_{k-512+\left\lfloor\frac{\Delta L}{2}\right\rfloor+L}, & 512-L-\left\lfloor\frac{\Delta L}{2}\right\rfloor \\
b_{k-512-\left\lceil\frac{\Delta L}{2}\right\rceil}, & 512+\left\lceil\frac{\Delta L}{2}\right\rceil \\
0, & \leq k<512+L+\left\lceil\frac{\Delta L}{2}\right\rceil \\
0 & \text { others }
\end{array}\right.
$$

where $\lceil\bullet\rceil$ and $\lfloor\bullet\rfloor$ denote the integer ceiling and floor operators, respectively, $L$ is the length of $a$ and $b$, whereas $\Delta L$ denotes the FD 


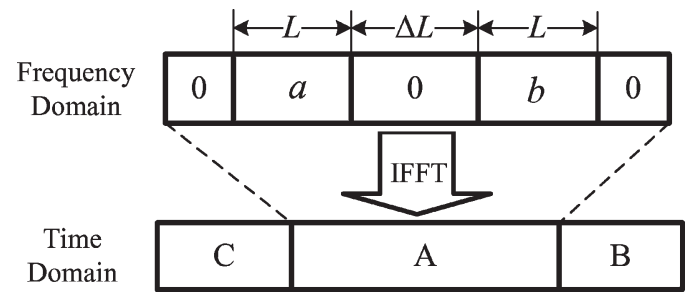

Fig. 2. Structure of the proposed preamble.

distance between $a$ and $b$, which varies according to the TPS requirements. The FD subcarrier symbols $\left\{X_{k}\right\}_{k=0}^{N-1}$ are then converted to the TD to create part "A" of the TD preamble by the $N$-point IFFT, as defined in (1).

The proposed preamble varies the distance between the pair of FD training sequences to create a signaling regime, where the FD distance may be varied across a wide range of values to satisfy the requirement of conveying 7-bit signaling. Taking a pair of length-255 training sequences, for example, the pair occupies two consecutive 255 subcarrier segments, which are allocated symmetrically from the center of the nominal bandwidth, as shown in Fig. 2. The value of $\Delta L$ is chosen to vary in the range of $[128,255]$ so that the required 7-bit signaling can be encoded into the 128 legitimate distance values. Further referring to the FD part in Fig. 2, the subcarriers at both ends are reserved so that the preamble can cope with large CFOs. The subcarriers in the center of the bandwidth are also reserved to reduce the impact of carrier leakage.

The performance associated with the proposed preamble depends on the chosen pair of FD training sequences. There are three main considerations in choosing these two training sequences: 1) good autocorrelation property; 2) simple correlator implementation; and 3) low peak-to-average power ratio (PAPR) in the TD after the IFFT operation. Pseudonoise (PN) sequences are widely used as training sequences since they have beneficial correlation properties. The optimal PN sequence may be found by searching through the entire PN sequence set [17] to ensure the lowest PAPR for the TD preamble. We suggest the pair $(a, b)$ to be the pair of identical DBPSK-modulated PN sequences, which have the lowest PAPR in the TD after the IFFT operation.

\section{B. Detection of the Proposed Preamble}

In the receiver, the GIC method in [12] is applied for timing and fractional CFO estimation. Afterwards, the TD part "A" is extracted from the preamble and converted to the FD by the $N$-point FFT operation, yielding

$$
Y_{k}=\frac{1}{\sqrt{N}} \sum_{n=0}^{N-1}\left(y_{n} e^{-j 2 \pi \hat{f}_{\mathrm{frc}} n}\right) e^{-j \frac{2 \pi}{N} n k}, \quad 0 \leq k<N
$$

where $\hat{f}_{\text {frc }}$ is the estimated fractional CFO, which is compensated in the TD before the FFT operation. The FFT result $\left\{Y_{k}\right\}_{k=0}^{N-1}$ is first differentially detected in the FD, i.e., $Y_{k} \cdot Y_{k-1}^{*}$, and then correlated with the local PN sequence $\left\{C_{k}\right\}_{k=0}^{L-1}$ to yield

$$
R_{l}=\frac{\sum_{k=0}^{L-1}\left(Y_{l+k} \cdot Y_{l+k-1}^{*}\right) \cdot C_{k}}{\frac{1}{2} \sum_{k=0}^{N-1}\left|Y_{k}\right|^{2}}, \quad 0 \leq l<N-L
$$

where $C_{k}$ is the sequence assigned to $a$ and $b$ before DBPSK modulation, whereas * denotes the complex conjugation. Since $\left\{C_{k}\right\}$ is a binary sequence, which assumes values from the set $\{-1,+1\}$, the

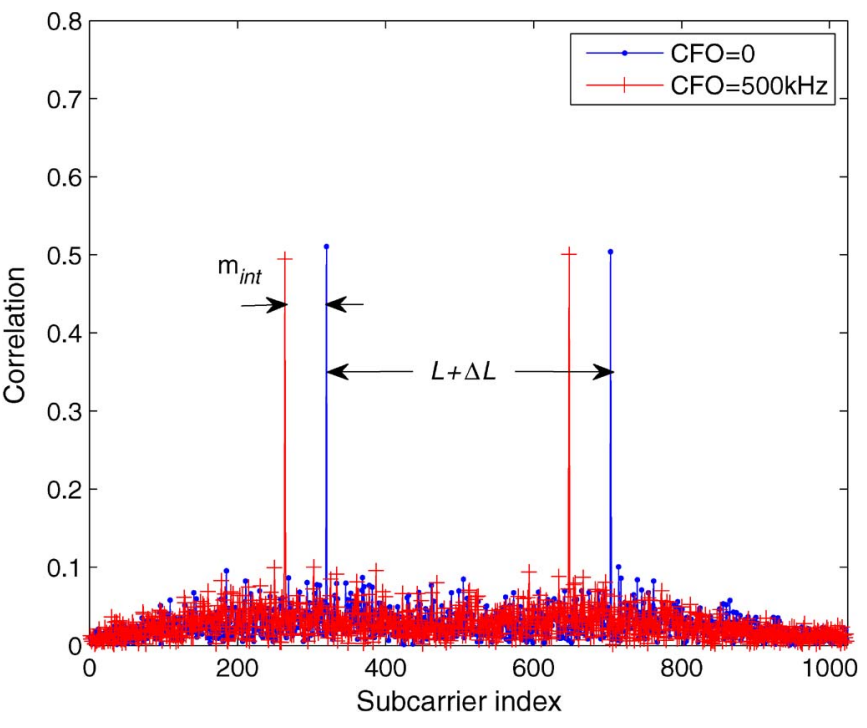

Fig. 3. Correlation results of the proposed preamble design in the AWGN channel given $\mathrm{SNR}=0 \mathrm{~dB}$.

multiplications by $C_{k}$ in the correlation (8) may be realized by a series of adders instead of multipliers. This significantly reduces the complexity of the proposed signaling detection algorithm.

Since there are two identical training sequences in $\left\{Y_{k}\right\}$, two peaks are expected in the correlation result in (8), and the distance between the two peaks gives an estimate of $\Delta L$ as

$$
\Delta \hat{L}=k_{2}-k_{1}-L
$$

where $k_{1}$ and $k_{2}$ are the correlation peak positions in the first and second half of $\left\{R_{k}\right\}$, respectively, namely

$$
\begin{aligned}
& k_{1}=\arg \max _{0 \leq l<\frac{N}{2}}\left|R_{l}\right| \\
& k_{2}=\arg \max _{\frac{N}{2} \leq l<N}\left|R_{l}\right| .
\end{aligned}
$$

The $\Delta \hat{L}$ value in (9) is then used to decode the 7-bit signaling information. The two peak positions also yield an estimate of the integer CFO, which is inferred from the peaks' shifts with their designed positions according to

$$
\hat{m}_{\text {int }}= \begin{cases}k_{1}-\left(512-\left\lfloor\frac{\Delta L+L}{2}\right\rfloor\right), & \text { if }\left|R_{k_{1}}\right| \geq\left|R_{k_{2}}\right| \\ k_{2}-\left(512+\left\lceil\frac{\Delta L+L}{2}\right\rceil\right), & \text { if }\left|R_{k_{1}}\right|<\left|R_{k_{2}}\right| .\end{cases}
$$

In contrast with the standardized P1 symbol, which requires a large number of correlations at the receiver for detecting the TPS, the proposed preamble design only requires a single correlator for detecting the TPS, hence yielding a considerable reduction in the receiver's complexity.

An example of the correlation function of (8) recorded for transmission over the AWGN channel at $\mathrm{SNR}=0 \mathrm{~dB}$ is shown in Fig. 3. We observe a shift of the correlation peak for $\mathrm{CFO}=500 \mathrm{kHz}$ from the reference position of $\mathrm{CFO}=0$. This shift gives a fine estimate of the integer CFO. It can also be seen from Fig. 3 that an accurate estimate of $\Delta L$ may be inferred from the distance between the two correlation peaks.

\section{Performance Evaluation}

We first analyze the theoretical performance of the proposed preamble design in the AWGN channel and then compare its signaling performance for transmission over frequency-selective fading channels to 


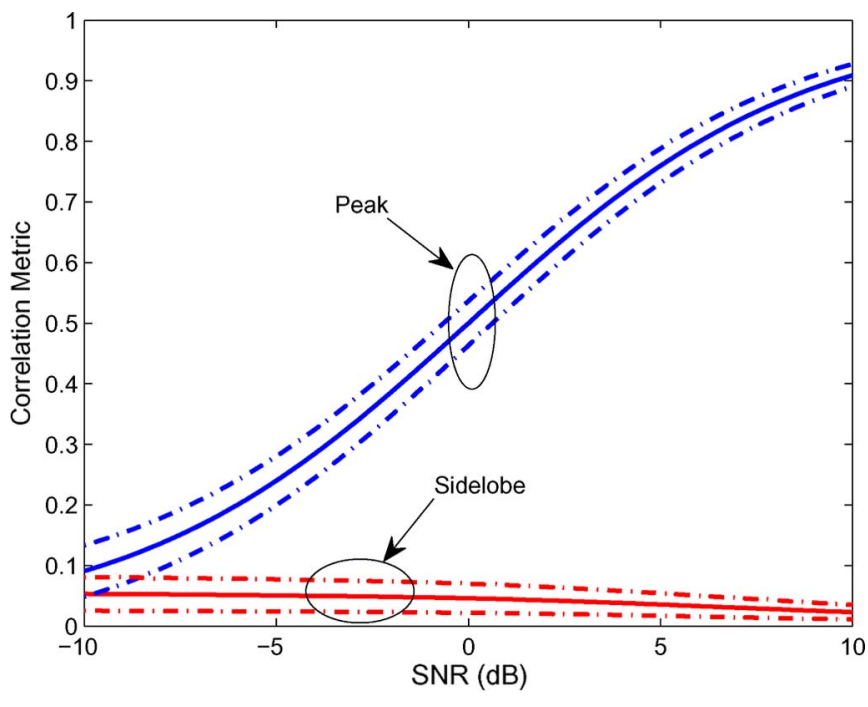

Fig. 4. Expectations of the correlation metric for the proposed preamble design in the AWGN channel given different SNR values, where the dashed lines indicate the standard deviations from the expectation.

that of the standardized P1 preamble using simulations. A comparison of the signaling detection complexity required by the two designs is also given.

\section{A. Theoretical Lower Bound Over AWGN Channel}

Assuming an ideal fraction CFO compensation in (7), the correlation peaks can be approximated by $R_{k_{1}}=X_{R}+\tilde{V}_{k_{1}}$ and $R_{k_{2}}=X_{R}+\tilde{V}_{k_{2}}$, where $X_{R}$ is real-valued, whereas $\tilde{V}_{k_{1}}$ and $\tilde{V}_{k_{2}}$ are complex-valued zero-mean random variables. Following a similar approach to that given in [10], we can conclude that the real and imaginary parts of both $R_{k_{1}}$ and $R_{k_{2}}$ have the Gaussian distributions $N\left(\mu_{R}, \sigma_{R}^{2}\right)$ and $N\left(0, \sigma_{R}^{2}\right)$, respectively, where $\mu_{R}$ and $\sigma_{R}^{2}$ are given by $[10]$

$$
\begin{aligned}
\mu_{R} & =\frac{\rho}{\rho+1} \\
\sigma_{R}^{2} & =\frac{\left(1+\mu_{R}^{2}\right) \rho+\left(2 L / N+\mu_{R}^{2}\right)}{N(\rho+1)^{2}} .
\end{aligned}
$$

Hence, the peak metrics $\left|R_{k_{1}}\right|$ and $\left|R_{k_{2}}\right|$ follow the same Rician distribution given by

$$
f_{\text {peak }}(y)=\frac{y}{\sigma_{R}^{2}} e^{-\frac{y^{2}+\mu_{R}^{2}}{2 \sigma_{R}^{2}}} I_{0}\left(\frac{\mu_{R} \cdot y}{\sigma_{R}^{2}}\right), \quad y>0
$$

where $I_{0}(\bullet)$ is the zero-order modified Bessel function of the first kind [18].

Assuming that the correlation of the training sequences is unity for perfect alignment and zero otherwise, both real and imaginary parts of the sidelobes follow the Gaussian distribution $N\left(0, \sigma_{R}^{2}\right)$. Thus, the sidelobe metric $\left|R_{k}\right|_{k \neq k_{1}, k_{2}}$ has a Rayleigh distribution given by

$$
f_{\text {side }}(y)=\frac{y}{\sigma_{R}^{2}} e^{-\frac{y^{2}}{2 \sigma_{R}^{2}}}, \quad y>0 .
$$

The expectations of the correlation peak and sidelobes at different SNRs are illustrated in Fig. 4, where the dashed lines indicate the standard deviations from the expectation. We can observe that for $\mathrm{SNR} \geq-5 \mathrm{~dB}$, the correlation metric can reliably separate the peak and the sidelobes.

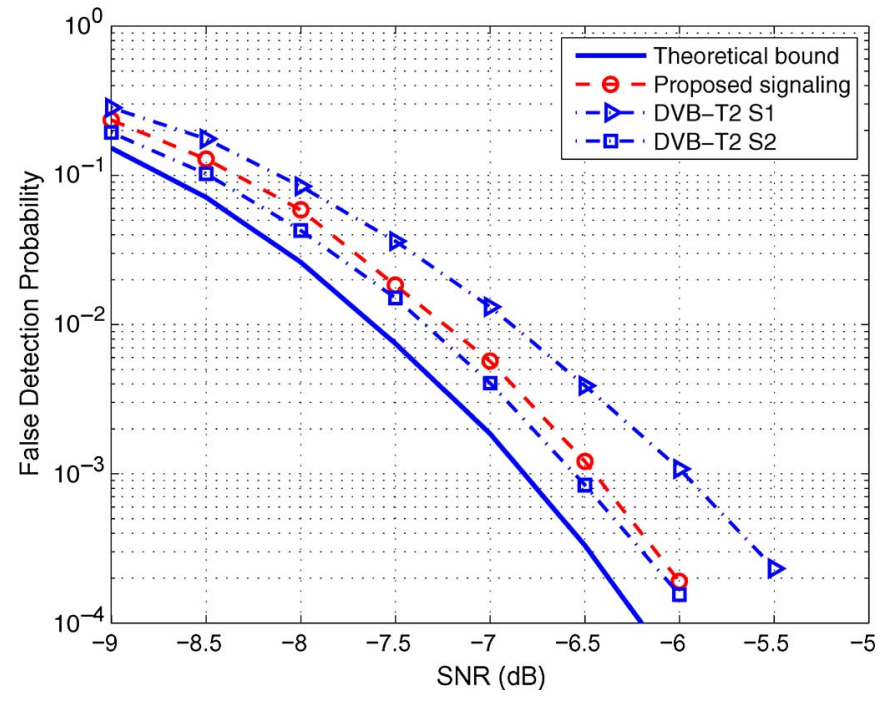

Fig. 5. False detection probabilities of the proposed signaling as well as the DVB-T2 S1 and S2 signaling over the AWGN channel in comparison with the theoretical bound.

Next, the probability of false detection is analyzed. Considering the first half of $\left\{R_{k}\right\}$, the probability that the sidelobe $\left|R_{k}\right|_{k \neq k_{1}}$ is higher than $\left|R_{k_{1}}\right|$ is given by

$$
P\left(\left|R_{k}\right|_{k \neq k_{1}}>\left|R_{k_{1}}\right|\right)=\int_{0}^{+\infty} \frac{y}{\sigma_{R}^{2}} e^{-\frac{2 y^{2}+\mu_{R}^{2}}{2 \sigma_{R}^{2}}} I_{0}\left(\frac{\mu_{R} \cdot y}{\sigma_{R}^{2}}\right) d y .
$$

The false peak detection probability is thus

$$
\begin{aligned}
P_{f} & =P\left(\left\{\max _{0 \leq k<\frac{N}{2}, k \neq k_{1}}\left|R_{k}\right|\right\}>\left|R_{k_{1}}\right|\right) \\
& =1-\left(1-P\left(\left|R_{k}\right|_{k \neq k_{1}}>\left|R_{k_{1}}\right|\right)\right)^{\frac{N}{2}-1} .
\end{aligned}
$$

If both correlation peaks are detected, then the estimation of $\Delta L$ and $m_{\text {int }}$ is deemed to be achieved. Thus, the false detection probability for $\Delta L$, which is denoted as $P_{\mathrm{FD}, \Delta L}$, and the false detection probability for $m_{\mathrm{int}}$, which is denoted as $P_{\mathrm{FD}, m_{\mathrm{int}}}$, are given by

$$
P_{\mathrm{FD}, \Delta L}=P_{\mathrm{FD}, m_{\mathrm{int}}}=1-\left(1-P_{f}\right)^{2}
$$

both of which are a function of the SNR.

The theoretical lower bound (19) is depicted in Fig. 5 in comparison with the performance of the proposed signaling detection obtained by simulation, which exhibited about $0.4-\mathrm{dB}$ degradation from the theoretical bound. The degradation of the actual signaling detection from the theoretical lower bound is mainly due to the following two factors. First, having a realistic timing recovery imposes some phase rotation after the FFT-based demodulation. Second, the residual fractional CFO imposes some ICI, which also results in an SNR loss (in decibels), quantified as [13]

$$
\mathrm{SNR}_{\text {loss }} \leq 10 \cdot \log \left(\frac{1+0.5947 \cdot \mathrm{SNR} \cdot(\sin \pi \varepsilon)^{2}}{(\sin \pi \varepsilon / \pi \varepsilon)^{2}}\right)
$$

where $\varepsilon=f_{\text {frc }}-\hat{f}_{\text {frc }}$ is the normalized residual CFO after the fractional CFO compensation.

The signaling detection performance of the standardized P1 symbol over the AWGN channel obtained by simulation is also given in Fig. 5 for comparison. Note that the S1 and S2 fields of the standardized P1 symbol have different priorities, whereas the 7-bit signaling of the 


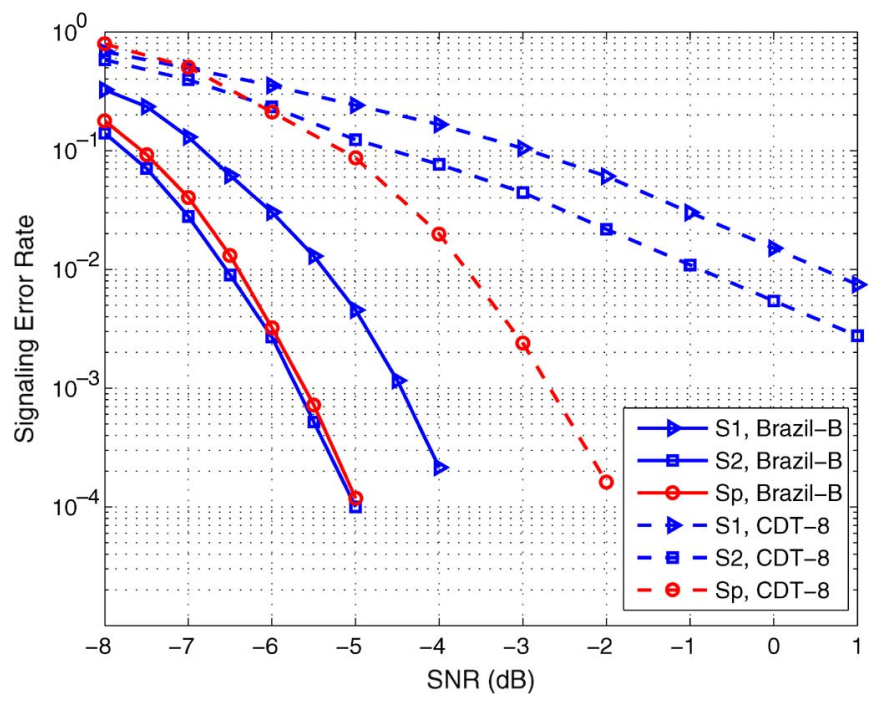

Fig. 6. SER comparison of the $\mathrm{S} 1$ and $\mathrm{S} 2$ signaling in the $\mathrm{P} 1$ preamble as well as the proposed signaling ( $\mathrm{Sp}$ ) over the Brazil-B and CDT-8 frequency-selective static channels.

proposed preamble has the same priority. It can be seen from Fig. 5 that the detection performance of the $\mathrm{S} 2$ signaling is marginally better than that of the proposed preamble, whereas the detection performance of the proposed signaling is much better than that of the S1 signaling. Thus, the signaling detection performance of the proposed preamble design is better than the average signaling detection performance of the standardized P1 symbol over the AWGN channel.

\section{B. Simulations Over Frequency-Selective Channels}

A simulation study was then carried out to compare the signaling detection performance of the proposed preamble with that of the standardized P1 symbol for transmission over frequency-selective channels. The same DVB-T2 transmission parameters were adopted, and the signal powers of both preambles were normalized in the simulation to ensure a fair comparison. The duration of both preambles was $224 \mu \mathrm{s}$ in the nominal 8-MHz system. The Brazil DTV field testing second channel model (Brazil-B) [4] and the China DTV testing eight channel model (CDT-8) [19] were adopted in the simulation. It should be noted that an unattenuated echo occurs in the CDT- 8 channel at a delay of $30 \mu \mathrm{s}$, which results in severe frequency selectivity. The signaling error rate (SER), which is defined as the false signaling detection probability, was evaluated.

Fig. 6 depicts the SER results obtained for the S1 and S2 signaling of the P1 preamble as well as for the proposed signaling (labeled as Sp) over the Brazil-B and CDT-8 static channels, whereas Fig. 7 compares the results for the Brazil-B and CDT- 8 fading channels with $50-\mathrm{Hz}$ Doppler frequency. For the Brazil-B channel whose frequency selectivity is not severe, it can be observed that the proposed signaling achieved a similar SER as the S2 signaling while outperforming the S1 signaling. It can also be seen from Figs. 6 and 7 that the proposed signaling achieved the performance gains by about 2.5 and $1.5 \mathrm{~dB}$, respectively, at the SER level of $10^{-2}$ over the severely frequencyselective CDT-8 static and fading channels. The reason for the much better signaling performance of the proposed preamble for transmission over severely frequency-selective channels can be explained as follows: Both the P1 symbol and the proposed design use differential decoding to alleviate the impact of the channel phase. The larger the phase difference for two adjacent subcarriers, the less effective this differential decoding. The frequency response of the CDT- 8 channel significantly varies from subcarrier to subcarrier owing to the severe

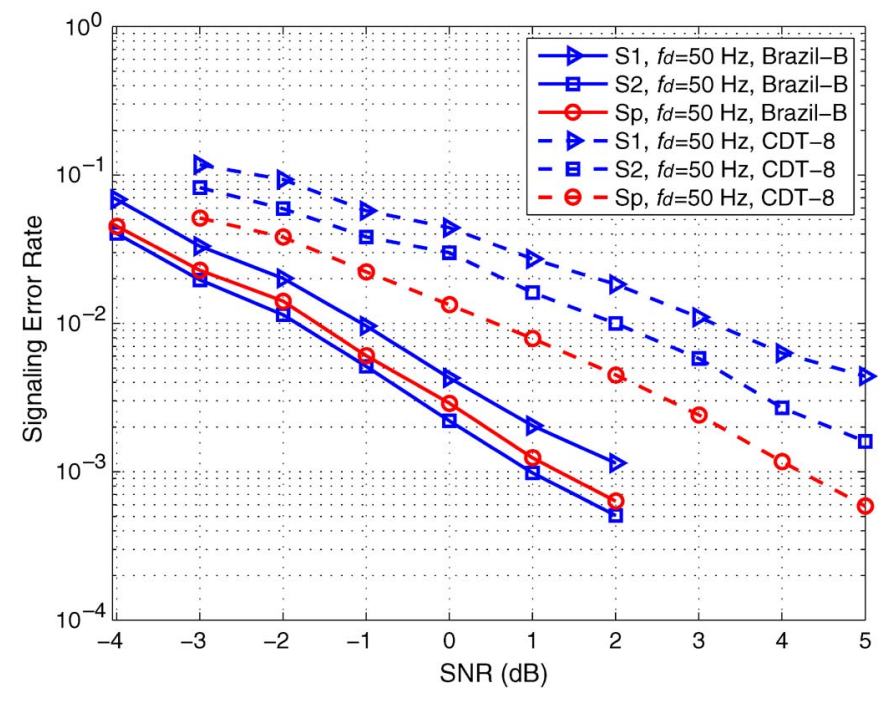

Fig. 7. SER comparison of the $\mathrm{S} 1$ and $\mathrm{S} 2$ signaling in the $\mathrm{P} 1$ preamble as well as the proposed signaling ( $\mathrm{Sp}$ ) over the Brazil-B and CDT-8 frequency-selective fading channels with 50-Hz Doppler frequency.

channel frequency selectivity. The active subcarriers in the standardized P1 symbol are randomly distributed in the FD, and the phases of two adjacent active subcarriers can differ significantly. In contrast, the phase difference of two adjacent subcarriers is much smaller for the proposed preamble, whose subcarriers are closely adjacent.

\section{Comparison of Computational Complexity}

As detailed in Section II, the detection of the P1 signaling in DVB-T2 includes four main steps: 1) the subcarrier pattern matching; 2) descrambling; 3) differential detection; and 4) correlation with the local CSS. In contrast, the proposed detection of the 7-bit signaling information only requires differential decoding and correlation with the local PN sequence. The computational complexity of the signaling detection algorithm may be quantified in terms of the number of multiplications and additions required. Assuming that the system is designed to cope with a maximum $\mathrm{CFO}$ of $\pm 500 \mathrm{kHz}$, i.e., up to \pm 56 subcarriers, the total number of computations imposed by detecting the standardized P1 signaling includes

$$
\begin{aligned}
384 & +56 \times 2+384 \times 2 \\
& =1264 \text { [complex-valued multiplications] } \\
384 & \times 56 \times 2+256 \times 128+64 \times 48 \\
& =78848 \text { [complex-valued additions] } .
\end{aligned}
$$

In contrast, the proposed signaling detection requires

$$
\begin{aligned}
255 & \times 2+56 \times 2+128 \\
& =750 \text { [complex-valued multiplications] } \\
255 & \times(56 \times 2+128) \\
& =61200 \text { [complex-valued additions]. }
\end{aligned}
$$

The proposed signaling detection method reduces the number of multiplications by about $40 \%$ and the number of additions by $20 \%$, in comparison to the standardized P1 symbol design. 


\section{CONCLUSION}

An improved preamble has been designed for signaling in OFDM broadcast systems, which exploits the presence of 128 possible distances between a pair of training sequences in the FD to infer the 7-bit signaling information. Only a single correlator is required at the receiver for signaling detection. Compared with the standardized P1 symbol of DVB-T2, the proposed design reduces the numbers of multiplications and additions by about $40 \%$ and $20 \%$, respectively. The simulation results have also shown that the proposed preamble achieves better signaling detection performance, in terms of false detection probability, than the standardized P1 preamble.

\section{REFERENCES}

[1] L. Hanzo, M. Münster, B. J. Choi, and T. Keller, OFDM and MC-CDMA for Broadband Multi-User Communications, WLANs and Broadcasting. Chichester, U.K.: Wiley, 2003.

[2] H. Sari and G. Karam, "Orthogonal frequency division multiple access and its application to CATV networks," Eur. Trans. Commun., vol. 45, pp. 507-516, Nov. 1998.

[3] M. Takada and M. Saito, "Transmission system for ISDB-T," Proc. IEEE, vol. 96, no. 1, pp. 251-256, Jan. 2006.

[4] J. Song, Z. X. Yang, L. Yang, K. Gong, C. Y. Pan, J. Wang, and Y. S. Wu, "Technical review on Chinese digital terrestrial television broadcasting standard and measurements on some working modes," IEEE Trans. Broadcast., vol. 53, no. 1, pp. 1-7, Mar. 2007.

[5] Frame Structure Channel Coding and Modulation for a Second Generation Digital Terrestrial Television Broadcasting System (DVB-T2), DVB Document A122, ETSI Std., Jun. 2008.

[6] IEEE Standard for Wireless LAN Medium Access Control (MAC) and Physical Layer (PHY) Specifications, IEEE 802.11, Nov. 1997.

[7] H. Ekstrom, A. Furuskar, J. Karlsson, M. Meyer, S. Parkvall, J. Torsner, and M. Wahlquist, "Technical solutions for the 3G longterm evolution," IEEE Commun. Mag., vol. 44, no. 3, pp. 38-45, Mar. 2006

[8] IEEE Standard for Local and Metropolitan Area Networks. Part 16: Air Interface for Fixed and Mobile Broadband Wireless Access Systems, IEEE 802.16e, Feb. 2006.

[9] F. Khan, LTE for 4G Mobile Broadband: Air Interface Technologies and Performance. Cambridge, U.K.: Cambridge Univ. Press, 2009.

[10] T. M. Schmidl and D. C. Cox, "Robust frequency and timing synchronization for OFDM," IEEE Trans. Commun., vol. 45, no. 12, pp. 1613-1621, Dec. 1997.

[11] F. Tufvesson, M. Faulkner, and O. Edfors, "Time and frequency synchronization for OFDM using PN sequence preambles," in Proc. VTC-Fall, Sep. 1999, vol. 4, pp. 2203-2207.

[12] J. G. Doblado, V. Baena, A. C. Oria, D. Perez-Calderon, and P. Lopez, "Coarse time synchronisation for DVB-T2," Electron. Lett., vol. 46, no. 11, pp. 797-799, May 2010.

[13] P. H. Moose, "A technique for orthogonal frequency division multiplexing frequency offset correction," IEEE Trans. Commun., vol. 42, no. 10, pp. 2908-2914, Oct. 1994.

[14] Implementation Guidelines for a Second Generation Digital Terrestrial Television Broadcasting System (DVB-T2), DVB Document A133, ETSI Std., Feb. 2009.

[15] C. C. Tseng and C. L. Liu, "Complementary sets of sequences," IEEE Trans. Inf. Theory, vol. IT-18, no. 5, pp. 644-652, Sep. 1972.

[16] C. D. Marziani, J. Urena, A. Hernandez, M. Mazo, F. J. Alvarez, J. J. Garcia, and P. Donato, "Modular architecture for efficient generation and correlation of complementary set of sequences," IEEE Trans. Signal Process., vol. 55, no. 2, pp. 2323-2337, May 2007.

[17] T. May, H. Rohling, and O. Edfors, "Reducing the peak-to-average power ratio in OFDM radio transmission systems," in Proc. VTC-Spring, May 1998, vol. 3, pp. 2474-2478.

[18] J. G. Proakis, Digital Communications, 3rd ed. New York: McGrawHill, 1995.

[19] F. Yang, J. T. Wang, J. Wang, J. Song, and Z. X. Yang, "Novel channel estimation method based on PN sequence reconstruction for Chinese DTTB system," IEEE Trans. Consum. Electron., vol. 54, no. 4, pp. 15831589, Nov. 2008.

\section{MIMO Precoding Using Rotating Codebooks}

Chengling Jiang, Michael Mao Wang, Chunliang Yang, Feng Shu, Jianxin Wang, Weixin Sheng, and Qian Chen

\begin{abstract}
Next-generation wireless communications rely on multiple-input-multiple-output (MIMO) techniques to achieve high data rates. Feedback of channel information can be used in MIMO precoding to fully activate the strongest channel modes and improve MIMO performance. Unfortunately, the bandwidth of the control channel by which the feedback is conveyed is severely limited. An important issue is how to improve the MIMO precoding performance with minimal feedback. In this paper, we present a method that uses a rotating codebook technique to effectively improve the precoding performance without the need to increase feedback overhead. The basic idea of the rotating codebook precoding is to expend the effective precoding codebook size via rotating multiple codebooks so that the number of feedback bits remains unchanged. Simulation results are presented to show the performance gain of the proposed rotating codebook precoding over conventional precoding.
\end{abstract}

Index Terms-Multiple-input-multiple-output (MIMO) precoding, quantized precoding.

\section{INTRODUCTION}

Multiple-input-multiple-output (MIMO) systems have become the most promising candidates for the next generation of high-data-rate wireless communications [1]. MIMO technology has been shown to provide significant system performance improvement over conventional systems by providing communication links with substantial diversity and capacity. This is especially true when channel state information (CSI) is available at the transmitter [2]. In fact, the closedloop capacity of a MIMO channel can be achieved by converting the channel into a set of parallel spatial layers via precoding and waterfilling power allocation at the transmitter and a linear minimum-meansquare-error (MMSE) filtering at the receiver wherein the optimal precoding and MMSE filter are determined by the singular value decomposition (SVD) of the MIMO channel matrix [3], [4].

For time-division-duplexing communication systems, where uplink and downlink channels share the same frequency band at different times, the downlink channel information can be estimated by the transmitter using the uplink pilot (analog feedback) due to the channel reciprocity property. For frequency-division-duplexing systems, where different frequency bands are allocated for the downlink and uplink channels, the channels are not reciprocal. Therefore, a CSI feedback channel, via the uplink control channel (digital feedback), is necessary to deliver the estimated channel knowledge back to the transmitter. For a time-varying channel, the channel knowledge at the transmitter should be regularly updated. The overhead, which linearly increases in the product of the number of antennas, the channel frequency selectivity, and the feedback frequency, can be large. Hence, the

Manuscript received July 6, 2010; revised October 15, 2010; accepted December 23, 2010. Date of publication January 28, 2011; date of current version March 21, 2011. The review of this paper was coordinated by Prof. W. A. Kryzmień.

The authors are with the Wireless Networking and Mobile Communications Group, School of Electronic and Optical Engineering, Nanjing University of Science and Technology, Nanjing 210094, China (e-mail: jiangchengling@mail.njust.edu.cn; wangmao@mail.njust.edu.cn; yclnjust@ 126.com; shufeng@mail.njust.edu.cn; wangjxin@mail.njust.edu.cn; chenq@ mail.njust.edu.cn).

Color versions of one or more of the figures in this paper are available online at http://ieeexplore.ieee.org.

Digital Object Identifier 10.1109/TVT.2011.2108323 